Artículo científico

Volumen 31(1):69-75. Enero-abril, 2020

e-ISSN 2215-3608, doi:10.15517/am.v31i1.37166

http://www.revistas.ucr.ac.cr/index.php/agromeso

\title{
Control químico de Pogonomyrmex barbatus con atrayentes de maíz, en Irapuato, Guanajuato, México ${ }^{1}$
}

\section{Chemical control of Pogonomyrmex barbatus with corn attractants, in Irapuato, Guanajuato, Mexico}

\author{
Manuel Darío Salas-Araiza², Luis Gerardo Lara-Álvarez², Oscar Alejandro Martínez-Jaime², \\ José Antonio Díaz-García ${ }^{3}$
}

1 Recepción: 16 de mayo, 2019. Aceptación: 7 de octubre, 2019. Este trabajo formó parte del proyecto "Evaluación de la eficacia de insecticidas en diferentes presentaciones sobre la población de Pogonomyrmex barbatus Smith (Hymenoptera: Formicidae)", financiado por la Compañía FMC México y se llevó a cabo en Irapuato Guanajuato, México.

2 Universidad de Guanajuato, Km. 9 carretera Irapuato-Silao, C.P. 36821, Irapuato, Guanajuato, México.lg.laraalvarez@ugto.mx, dariosalasaraiza@hotmail.com,oscarja@ugto.mx (https://orcid.org/0000-0001-8328-5686).

3 Universidad Autónoma de Chihuahua, Facultad de Zootecnia y Ecología, Periférico Francisco R. Almada Km. 1, Col. Zootecnia, C.P. 33820 , Chihuahua, Chihuahua, México.jadiaz@uach.mx

\section{Resumen}

Introducción. Las hormigas granívoras tienen impacto económico y ecológico al ser consideradas plagas agrícolas que transportan semillas y defolian plantas cultivadas. Objetivo. El objetivo de este trabajo fue evaluar la efectividad de los insecticidas Imidacloprid y Novalurón en mezcla, con tres atrayentes a base de harina de maíz para el control de Pogonomyrmex barbatus, en pruebas de campo en Irapuato, Guanajuato, México. Materiales y métodos. Esta investigación se realizó de agosto a noviembre del 2014, se consideraron seis tratamientos, los tres primeros consistieron de la mezcla de Imidacloprid con harina de maíz (en pellet molido fino, en pellet molido y sin pelletizar), el cuarto de Novalurón combinado con harina de maíz en pellet molido, y los últimos dos constaron solamente de harina de maíz (en pellet molido fino y sin pelletizar), además de un testigo absoluto. Se realizaron conteos de individuos por minuto antes (lectura a 0 h) y después de la aplicación de los tratamientos (lecturas a 1, 24, 72, 144 y 240 h) en el exterior de tres hormigueros seleccionados previamente para tal fin. Se realizó el análisis de varianza del diseño completamente al azar con arreglo en franjas sub-subdivididas con tres repeticiones, y tres factores que fueron los siguientes: seis tratamientos y el testigo absoluto, seis lecturas a través del tiempo incluyendo el conteo previo a la aplicación de los tratamientos y los tres hormigueros. Resultados. Se presentaron diferencias estadísticas altamente significativas para los factores: tratamientos y lecturas, así como para la interacción entre ambos. Conclusión. Los tratamientos con insecticida mostraron su máximo control a las $72 \mathrm{~h}$ de su aplicación, los mejores fueron el Imidacloprid combinado con harina de maíz en dos presentaciones (en pellet molido y sin pelletizar), con mayor eficacia a partir de las 24 h de su aplicación.

Palabras clave: cebo para control de plagas, hormigas, Imidacloprid, benzoilfenilureas, harina de maíz. 


\begin{abstract}
Introduction. Granivorous ants have an economic and ecological impact because they are considered agricultural pests that transport seeds and defoliate cultivated plants. Objective. The objective of this work was to evaluate the effectiveness of the insecticides Imidacloprid and Novaluron in mixture with three cornflour-based attractants for the control of Pogonomyrmex barbatus in field trials in Irapuato, Guanajuato, Mexico. Materials and methods. This research was conducted from August to November 2014, six tratments were considered, the first three consisted of the mixture of Imidacloprid with cornflour (in finely milled pellet, in milled pellet and without pelletizing), the fourth was Novaluron combined with cornflour in milled pellet, and the last two consisted only of cornflour (in finely milled pellet and without pelletizing), in addition to an absolute control. Individuals counts per minute were performed before (reading at $0 \mathrm{~h}$ ), and after the application of the treatments (readings at 1,24,72, 144, and $240 \mathrm{~h}$ ) on the outside of three anthills previously selected for this purpose. The analysis of variance of the design was completely randomized according to sub-subdivided stripes with three repetitions, and three factors that were the following: six treatments and the absolute control, six readings over time including the count prior to the application of the treatments and the three anthills. Results. There were highly significant statistical differences for the factors: treatments and readings, as well as for the interaction between both. Conclusion. The treatments with insecticide showed their maximum control at $72 \mathrm{~h}$ of its application, the best were the Imidacloprid combined with cornflour in two presentations (in milled pellet and without pelletizing), showing greater effectiveness after $24 \mathrm{~h}$ of its application.
\end{abstract}

Keywords: pest control baits, ants, Imidacloprid, benzoylphenylureas, corn flour.

\title{
Introducción
}

Las hormigas granívoras emplean las semillas como su principal fuente de alimento y las almacenan en galerías dentro del nido para su posterior consumo (Rojas, 2001; Pirk, 2002). En el mundo se han identificado más de 150 especies de hormigas granívoras dentro de dieciocho géneros (McMahon et al., 2000), pertenecientes a las subfamilias Ponerinae, Formicinae y Myrmicinae, destacando esta última por contar con 35 especies con este comportamiento (Hölldobler y Wilson, 1990), las cuales comen y dispersan semillas en la mayoría de los ecosistemas terrestres, excepto en climas extremos como el de la Antártida (Greene y Gordon, 2007).

Las hormigas granívoras tienen impacto económico y ecológico, ya que son plagas en áreas agrícolas, al transportar semillas de plantas no deseables en campos de cultivo y además, defoliar las plantas cultivadas (Guzmán-Mendoza et al., 2012). Pogonomyrmex barbatus (Smith, 1858) (Formicidae, Myrmicinae) es una hormiga granívora distribuida ampliamente en el centro de México, que construye nidos subterráneos que provocan la pérdida de agua de riego, dificultan el paso de la maquinaria y minan los bordos de almacenamiento de agua (Johnson, 2000). Las estrategias modernas de manejo y regulación de estos insectos contemplan el uso de cebos, por ser un método seguro, específico y eficaz para controlar sus colonias, y sin la necesidad de identificar los nidos (Suiter et al., 1997).

Los cebos utilizados son formulados a base de mezclas de atrayentes alimenticios e insecticidas, de manera que inducen a las hormigas a llevar estas sustancias al interior del nido, lo que incrementa la efectividad en el control; al respecto Anda-Rickert y Fracchia (2012) señalaron que el eleosoma de diversas especies vegetales tiene una gran semejanza con los ácidos grasos de 16 y 18 carbonos de ciertos insectos, que complementan la dieta de Pogonomyrmex, lo que desencadena una respuesta de transporte de las semillas hasta las cámaras más profundas del nido donde se encuentran las crías y las obreras jóvenes. Debido a la variedad en las preferencias alimenticias 
de cada especie de hormiga y a su comportamiento, es conveniente determinar cuál atrayente es el más adecuado en cada especie para integrarlo a los cebos.

El objetivo del presente trabajo fue evaluar la efectividad de los insecticidas insecticidas Imidacloprid combinado con tres atrayentes a base de harina de maíz, para el control de P. barbatus, en pruebas de campo en Irapuato, Guanajuato, México.

\section{Materiales y métodos}

El presente estudio se realizó entre agosto y noviembre del 2014 en el campo experimental del Departamento de Agronomía de la División de Ciencias de la Vida, ubicado en el municipio de Irapuato, Guanajuato (2040’06" N y $101^{\circ} 20^{\prime} 14$ " O), a una altura de $1729 \mathrm{msnm}$, con temperatura media anual de $20,3{ }^{\circ} \mathrm{C}$ y una precipitación pluvial anual de 675,1 mm (Servicio Meteorológico Nacional, 2015). Para efectuar el ensayo, se identificaron tres hormigueros dentro del campo experimental, separados aproximadamente a $500 \mathrm{~m}$ uno del otro, en cada hormiguero se realizó un ensayo que incluyó seis tratamientos y un testigo absoluto, seis lecturas (conteos de hormigas por minuto) en diferentes intervalos de tiempo y tres repeticiones. En cada hormiguero, se determinó la ruta con mayor actividad de hormigas forrajeras y se seleccionó una superficie de 30x30 cm con la ayuda de estacas de madera de $15 \mathrm{~cm}$ de altura, a las estacas se sujetó un hilo de algodón para delimitar el área de medición.

Para cuantificar la actividad externa de cada hormiguero, se contabilizó el número de hormigas que pasaron por la zona delimitada con rumbo a la entrada del hormiguero en el lapso de un minuto, se realizaron tres conteos con intervalos de dos minutos entre cada uno, entre las 10:30 y las 12:30 h que es cuando la actividad de las hormigas es mayor. Estas mediciones se hicieron antes de administrar los tratamientos $(0 \mathrm{~h})$ y después de su aplicación en cinco registros $(1,24,72,144$ y 240 h). Los tratamientos estuvieron conformados por combinaciones de insecticidas y atrayentes con diferentes niveles de granulometría; se agregaron dos tratamientos de referencia (solo con atrayente) y un testigo absoluto (Cuadro 1). Para evaluar el efecto de los insecticidas a través del tiempo sobre la cinética de la colonia de $P$. barbatus, se aplicaron $20 \mathrm{~g}$ de la mezcla de cada tratamiento distribuidos a 1,5 $\mathrm{m}$ de distancia de la entrada del hormiguero.

Cuadro 1. Lista de los tratamientos a base de insecticidas y harina de maíz, evaluados para controlar la hormiga Pogonomyrmex barbatus. Campo experimental del Departamento de Agronomía de la División de Ciencias de la Vida, Irapuato, Guanajuato, México. 2014.

Table 1. List of the treatments based on insecticides and corn flour evaluated to control the ant Pogonomyrmex barbatus. Experimental Field of the Agronomy Department of the Life Sciences Division, Irapuato, Guanajuato, Mexico. 2014.

\begin{tabular}{ccccc}
\hline Número & Tratamiento & Ingrediente activo & Atrayentes alimenticios & Granulometría (mm) \\
\hline 1 & Dardo_polvo & Imidacloprid & Harina de maíz en pellet molido fino & $0-1$ \\
2 & Dardo_molido & Imidacloprid & Harina de maíz en pellet molido & $1,2-2$ \\
3 & Dardo_harina & Imidacloprid & Harina de maíz sin pelletizar & $<0.5$ \\
4 & Novalurón & Novalurón & Harina de maíz en pellet molido & $5-10$ \\
5 & Pellet_molido & -------------- & Harina de maíz en pellet molido fino & $0-1$ \\
6 & Harina_maíz & --------------- & Harina de maíz sin pelletizar & $<0,5$ \\
7 & Testigo & -------------- & Sin aplicación & \\
\hline
\end{tabular}

La aplicación de los tratamientos se realizó cuando el número de hormigas por minuto varió entre 30 y 35 . Para analizar la variable de respuesta que fue el número de hormigas por minuto (nhpm), se utilizó un diseño completamente 
aleatorio con arreglo en franjas sub-subdivididas con tres repeticiones, donde los factores fueron los tratamientos (TRAT=7); las lecturas a través del tiempo (LECT=6) incluyeron la efectuada antes de aplicar los tratamientos $(0 \mathrm{~h})$ y los hormigueros $(\mathrm{HORM}=3)$. Dado que la variable dependiente nhpm es una variable de conteo, para poder llevar a cabo el análisis de varianza correspondiente, se realizó la transformación, donde nhpmt es el número de hormigas por minuto transformada, la cual cumplió los supuestos del análisis de varianza (Anderson y McLean, 1974; Montgomery, 2013), una vez realizado este último se concluyó rechazar la hipótesis nula, por lo que se procedió a aplicar la prueba de comparación del rango múltiple de Duncan, con un nivel de significancia de p=0,05. El análisis de la información, transformación de la variable nhpm, análisis de varianza para el diseño planteado, prueba de comparación múltiple y gráficas, se realizaron usando el paquete de programación R (R Development Core Team, 2008).

\section{Resultados}

Los tratamientos (TRAT) presentaron diferencias altamente significativas $\left(\mathrm{F}^{6}{ }_{14}=7,17 ; \mathrm{P}=0,0012 * *\right)$, así como el periodo de efectividad de los productos (LECT) $\left(\mathrm{F}_{58}^{30}=12,09 ; \mathrm{P}=0,0002^{* *}\right)$ y la interacción entre ambos (TRAT*LECT) $\left(\mathrm{F}_{12}^{5}=6,92 ; \mathrm{P}=0,0001 * *\right)$.

Los tratamientos Dardo_molido y Dardo_harina fueron los más efectivos (Duncan p=0,05) para disminuir la actividad de las hormigas $P$. barbatus, al presentar el menor número promedio con 15 y 30 hormigas por minuto, respectivamente (Figura 1).

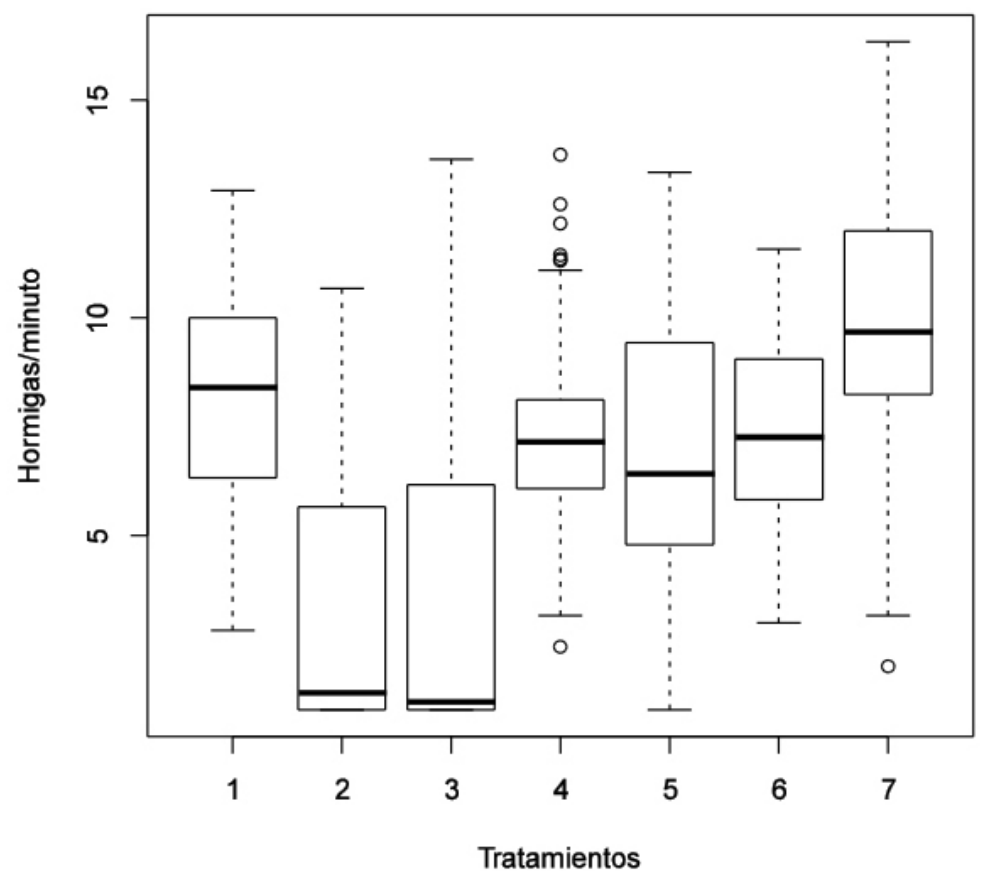

Figura 1. Efecto de los tratamientos con insecticidas Imidacloprid (Dardo): Dardo_polvo, Dardo_molido, Dardo_harina y Novalurón, y de los cebos (harina de maíz en pellet molido o molido fino o sin peletizar), en las poblaciones de P. barbatus. Prueba del Rango Múltiple de Duncan $(\mathrm{p}=0,05)$ del factor tratamiento (TRAT) para la variable nhpmt. Se reportan las medias de la variable transformada nhpmt. Campo experimental del Departamento de Agronomía de la División de Ciencias de la Vida, Irapuato, Guanajuato, México. 2014.

Figure 1. Effect of treatments with insecticides Imidacloprid (Dardo): Dardo_dust, Dardo_milled, Dardo_flour and Novaluron, and of the baits (cornflour in milled pellet or finely milled pellet or without pelletizing), on the populations of $P$. barbatus. Duncan Multiple Range Test $(\mathrm{p}=0.05)$ of the treatment factor (TRAT) for the variable nhpmt. The means of the transformed variable nhpm are reported. Experimental Field of the Agronomy Department of the Life Sciences Division, Irapuato, Guanajuato, Mexico. 2014. 
Cuando se administró la combinación Dardo_molido, la mayor parte de las hormigas exploraron este compuesto, pero después lo ignoraron, se registró mortandad sobre el camino donde circularon e incluso sobre el producto aplicado a las $24 \mathrm{~h}$, y se observó que las hormigas trasladaron los cadáveres lejos del nido. Con el tratamiento Dardo_harina se presentó una actividad mínima, con hormigas paralizadas sobre el camino, aunque no se observó cambio de ruta sí se notó la extracción de cadáveres del nido, pero en menor grado que con el cebo Dardo_molido.

Los tratamientos Dardo_molido y Dardo_harina, disminuyeron el número de hormigas una hora después de su aplicación, hasta cero individuos a las 72 h (Figura 2). Con el cebo formulado con Dardo_polvo la población inició su descenso a las $24 \mathrm{~h}$ y alcanzó su mínimo a las $72 \mathrm{~h}$, posteriormente se incrementó hasta acercarse al número original de hormigas.

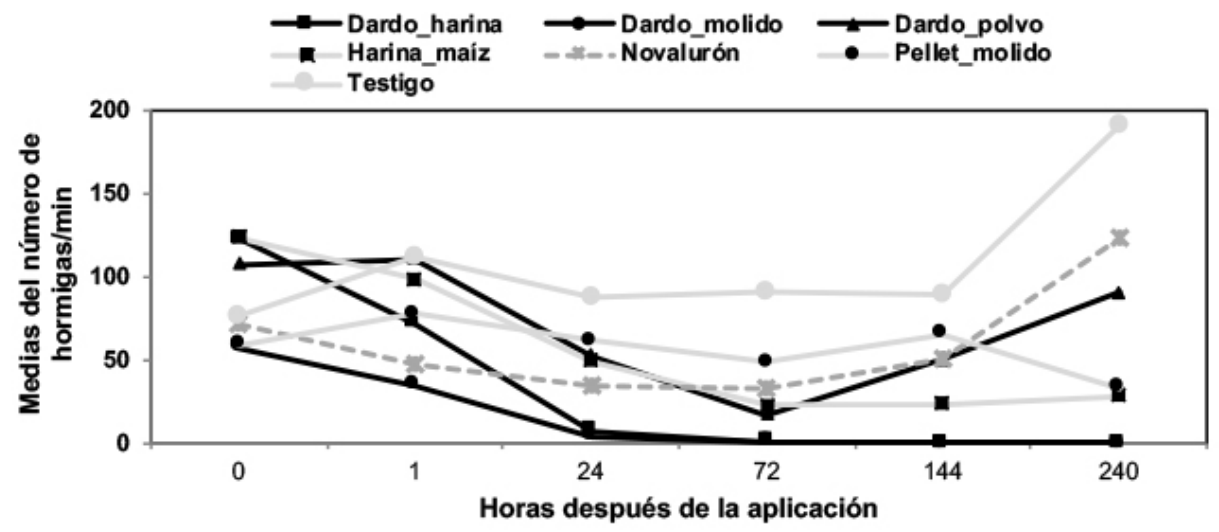

Figura 2. Promedio de hormigas (Pogonomyrmex barbatus (Smith)) por minuto registradas en los diferentes tratamientos (insecticidas: Imidacloprid y Novalurón, y como atrayente alimenticio harina de maíz en pellet molido o molido fino o sin peletizar) a lo largo de las seis lecturas. Se registran las medias de la variable original: hormigas por minuto para la interacción: tratamiento*lectura. Campo experimental del Departamento de Agronomía de la División de Ciencias de la Vida, Irapuato, Guanajuato, México. 2014.

Figure 2. Average of ants (Pogonomyrmex barbatus (Smith)) per minute recorded in the different treatments (insecticides: Imidacloprid and Novaluron and as a food attractant cornflour in milled pellet or finely milled pellet or without pelletizing) throughout the six readings. The means of the original variable are recorded: ants per minute for the interaction: treatment*reading. Experimental Field of the Agronomy Department of the Life Sciences Division, Irapuato, Guanajuato, Mexico. 2014.

\section{Discusión}

El ingrediente activo de Dardo_polvo y Dardo_molido fue Imidacloprid, este neonicotenoide actúa por contacto e ingestión en los insectos interfiriendo en las señales nerviosas de las neuronas, y ocasiona parálisis y convulsiones casi de inmediato, por esta razón a las $72 \mathrm{~h}$ las poblaciones disminuyeron a cero con Dardo_harina; Basnet et al. (2016) reportaron el mismo efecto a las $24 \mathrm{~h}$ después de aplicar un neonicotenoide contra cinco especies de hormigas en áreas urbanas. Para el tratamiento Dardo_polvo, a pesar de contener el mismo ingrediente activo, se observó que el efecto sobre los formícidos disminuyó (Figura 1), ya que mostró una media de setenta hormigas por minuto, ligeramente más alta pero estadísticamente igual a las que mostraron el Novalurón y los tratamientos con atrayente sin insecticida, que fueron de alrededor de sesenta individuos (Figura 2); la presentación en polvo fue acarreada al interior del hormiguero formando una esfera, lo cual requiere de mayor trabajo, de ahí su posible poca atracción.

El proceso de acarreo e introducción de cada uno de los tratamientos al interior del hormiguero tardó alrededor de una hora, al cabo de $24 \mathrm{~h}$ de la aplicación los cebos fueron llevados nuevamente al exterior del hormiguero. 
Esta ligera disminución del número de hormigas a los 60 min de la aplicación, se debe posiblemente a un efecto de repelencia al cebo y no al efecto del ingrediente activo en el proceso de muda, ya que la población inició su recuperación al final del ensayo $(240$ h), el mismo efecto de restablecimiento poblacional fue reportado por Hara y Niino-DuPonte (2017) en el control de la hormiga Wasmannia auropunctata (Roger), utilizando el ingrediente activo Pyriproxifen.

Cuando se aplicó el tratamiento Dardo_harina, las hormigas identificaron este cebo de inmediato y comenzaron a llevarlo al nido, formando pequeñas esferas con la harina y acarreándolas con el psamóforo que tienen en la parte inferior de su aparato bucal; la misma reacción sobre los insectos fue provocada por el tratamiento Novalurón (combinado con harina de maíz en pellet molido). En ambos tratamientos también se percibió actividad de reclutamiento de otras hormigas forrajeras; en este sentido, Helms y Gardner (2013), indicaron que esta acción de incorporación de hormigas del mismo nido ocurre de manera espontánea, sobre todo en el proceso inicial de formación del grupo. Por su parte, Greene et al. (2013) manifestaron que cuando los individuos de Pogonomyrmex barbatus regresan al nido con semillas, estimulan el inicio de la actividad de las forrajeras inactivas dentro de las galerías, ya que responden al olor de las que llegan y de las semillas que acarrean.

Los tratamientos donde se combinaron los atrayentes con Imidacloprid afectaron la actividad de las diferentes hormigas de $P$. barbatus del nido, las hormigas exploradoras alertan a otras sobre la presencia de alimento y estas cambian de actividad para ayudar en el acarreo de forraje (Barton et al., 2002), asimismo, es posible que al detectar la presencia de un alimento conocido como los usados en los cebos, una mayor población llevó este compuesto al interior del hormiguero y el resultado fue mejor al reducir la densidad poblacional.

\section{Conclusiones}

El mejor tratamiento fue Dardo_molido que contenía Imidacloprid, el cual disminuyó considerablemente la actividad externa de $P$. barbatus, a partir de las 24 h de su aplicación ( 3,9 hormigas por minuto en promedio). Todos los tratamientos mostraron su mayor eficacia a las $72 \mathrm{~h}$. El Novalurón fue el producto menos eficaz.

\section{Literatura citada}

Anda-Rickert, A., y S. Fracchia. 2012. La biología de Pogonomyrmex cunicularius pencosensis (Hymenoptera: Formicidae) en relación con su comportamiento como dispersora de semillas con eleosoma en el Noroeste semiárido argentino. Rev. Soc. Entomol. Argent. 71(1-2):11-27.

Anderson, V.L., and R.A. McLean. 1974. Design of experiments: A realistic approach. Marcel Dekker Inc., NY, USA.

Barton, K.E., N.J. Sanders, and D.M. Gordon. 2002. The effects of proximity and colony age on interspecific interference competition between the desert ants Pogonomyrmex barbatus and Aphaenogaster cockerelli. Am. Midl. Nat. 148:376382. doi:10.1674/0003-0031(2002)148[0376:TEOPAC]2.0.CO;2

Basnet, S., D. Richman, R.B. Narain, and S.T. Kamble. 2016. Efficacy of transport mikron against nuisance ants when applied around structures. Arthropod. Manag. Tests 41:tsw018. doi:101093/amt/tsw018

Greene, M.J., and D.M. Gordon. 2007. Interaction rate informs harvester ant task decisions. Behav. Ecol. 18:451-455. doi:10.1093/beheco/arl105

Greene, M.J., N.W. Pinter, and D.M. Gordon. 2013. Interactions with combined chemical cues inform harvester ant foragers' decisions to leave the nest in search of food. PLos ONE 8(1):e522019. doi:10.1371/journal.pone.0052219 
Guzmán-Mendoza, R., G. Castaño-Meneses, and J.A. Zavala-Hurtado. 2012. Foraging activity and trophic spectrum of red ant Pogonomyrmex barbatus Smith, 1858, in productivity-contrasted microenvironments. Psyche. J. Entomol. 2012:942737. doi:10.1155/2012/942737

Hara, A.H., and R. Niino-DuPonte. 2017. Attractancy of two Pyriproxyfen ant baits to control the little fire ant. Arthropod. Manag. Tests 42(1):tsw144. doi:10.1093/amt/tsw144

Helms, C.S., and E.M. Gardner. 2013. The emergence of reproductive division of labor in forced queen groups of the ant Pogonomyrmex barbatus. J. Zool. 291:12-22. doi:10.1111/jzo.12071

Hölldobler B., and E.O. Wilson. 1990. The ants. Harvard University Press, Cambridge, MA, USA.

Johnson, R.A. 2000. Habitat segregation base on soil texture and body size in the seed-harvester ants Pogonomyrmex rugosus and P. barbatus. Ecol. Entomol. 25:403-412. doi:10.1046/j.1365-2311.2000.00286.x

McMahon, J., J.F. Mull, and T.O. Crist. 2000. Harvester ants (Pogonomyrmex spp.): Their community and ecosystem influences. Annu. Rev. Ecol. Syst. 31:265-291. doi:10.1146/annurev.ecolsys.31.1.265

Montgomery, D.C. 2013. Design and analysis of experiments. John Wiley \& Sons Inc., NY, USA.

Pirk, G.I. 2002. Dieta de las hormigas granívoras Pogonomyrmex pronotalis y Pogonomyrmex rastratus en el Monte Central. Tesis Lic., Universidad de Buenos Aires, Buenos Aires, ARG.

R Development Core Team. 2008. R: A language and environment for statistical computing. R Foundation for Statistical Computing, Vienna, AUT. http://www.R-project.org (accessed Aug. 22, 2017).

Rojas, F.P. 2001. Las hormigas del suelo en México: Diversidad, distribución e importancia (Hymenoptera: Formicidae). Acta Zool. Mex. (n.s.). 1(esp.):189-238.

Servicio Meteorológico Nacional. 2015. Normales climatológicas. Irapuato, Guanajuato, México. Servicio Metereológico Nacional, MEX. https://smn.conagua.gob.mx/tools/RESOURCES/Normales5110/NORMAL11028.TXT (consultado 23 nov. 2017).

Suiter, D., D. Wu, and G. Bennett. 1997. The evolution of ant control. Pest Control 65:46-51. 